\title{
Green synthesis of Microalgal biomass-silver nanoparticles composite showing antimicrobial activity and heterogenous catalysis of nitrophenol reduction
}

\section{Sushree S. Priyadarshini}

CSIR-IMMT: Council of Scientific and Industrial Research Institute of Minerals and Materials Technology

Shradhanjali Sethi

CSIR-IMMT: Council of Scientific and Industrial Research Institute of Minerals and Materials Technology

\section{Shweta Rout}

CSIR-IMMT: Council of Scientific and Industrial Research Institute of Minerals and Materials Technology Pravat Manjari Mishra

CSIR-IMMT: Council of Scientific and Industrial Research Institute of Minerals and Materials Technology Nilotpala Pradhan ( $\square$ npradhan@immt.res.in )

CSIR-IMMT: Council of Scientific and Industrial Research Institute of Minerals and Materials Technology

\section{Research Article}

Keywords: Microalgae, Scenedesmus, Silver Nanoparticle, p-Nitrophenol, Heterogenous Catalysis, Antimicrobial activity

Posted Date: April 8th, 2021

DOl: https://doi.org/10.21203/rs.3.rs-398850/v1

License: (c) (i) This work is licensed under a Creative Commons Attribution 4.0 International License.

Read Full License 


\section{Abstract}

In this study we have demonstrated an integrated approach for utilization of microalga Scenedesmus sp, generated during $\mathrm{CO}_{2}$ sequestration, in fabrication of catalytic and antimicrobial silver nanoparticles composite. The algal biomass was grown in open raceway pond of $30,000 \mathrm{~L}$ scale for $\mathrm{CO}_{2}$ sequestration, after which it was harvested and dried. The dried biomass served as a reducing and immobilizing agent for silver nanoparticles. The biomass-silver nanoparticles composite was used for the first time, as a heterogenous catalyst for reduction of a prominent industrial pollutant, $\mathrm{p}$-Nitrophenol, in aqueous medium under ambient condition. Series of experiments were conducted to evaluate the effect of calcination temperature, load and reuse of the material on its catalytic efficiency. The calcined composite material was found to be an excellent catalyst for reduction of $\mathrm{p}$-Nitrophenol. As low as $5 \mathrm{mg} \mathrm{mL}^{-1}$ calcined material could catalyse the reduction of more than $95 \% \mathrm{p}$-Nitrophenol at the rate of $0.60 \mathrm{mg} \mathrm{L}^{-1}$ $\min ^{-1}$ in aqueous solution at $10 \mathrm{ppm}$ concentration. The efficiency of catalysis was evaluated for ten cycles of reuse. The material was found to be a potential candidate as a reusable heterogenous catalyst for designing continuous flow system for remediation of industrial effluents rich in p-Nitrophenol. Furthermore the material served as a good antimicrobial agent against pathogenic bacteria and fungi. Two strains each of gram + ve and gram - ve bacteria and three strains of fungi were used in the antimicrobial studies using well diffusion method.

\section{Introduction}

Concern for environmental pollution has risen in parallel to the rapid progress in industrialisation, with air and water pollution particularly demanding immediate mitigation strategies. In this regard, nanotechnology provides tremendous opportunity in development of efficient tools for elimination of diverse pollutants from the environment. Nanoparticles synthesized using biological entities such as plants, algae, fungi and bacteria are greener and cheaper alternative to chemical synthesis $[1,2,3,4]$. One of the fascinating sources for such nanoparticles is microalgae. Microalgae are unicellular, photosynthetic algae with remarkable capacity for $\mathrm{CO}_{2}$ fixation [5]. They are a renewable and sustainable source of biofuels, neutraceuticals, cosmetics and food ingredients [6]. In the past few years microalgae have emerged as an eco-friendly source for green synthesis of various nanoparticles with potential application in environmental mitigation $[7,8,9]$.

Scenedesmus is a common freshwater microalga. It has high biomass productivity as compared to other microalgae and is easy to grow $[10,11,12]$. Due to high efficiency in $\mathrm{CO}_{2}$ capture, many reports show its utilisation for sequestration of gaseous $\mathrm{CO}_{2}$ emitted by industries like coal-based power-plants and steel industry $[13,14,15,16,17]$. Cell-free extracts and live biomass of Scenedesmus have been reported for nanoparticles synthesis $[18,19]$. In the current investigation, we report for the first time, as-harvested dry biomass of Scenedesmus for synthesis as well as immobilization of silver nanoparticles. Once synthesized, the silver nanoparticles adhered to the biomass which together formed a biomassnanoparticle composite. Calcination of the composite led to the formation of a solid, robust and reusable 
catalyst for reduction of $\mathrm{p}$-Nitrophenol (PNP) in the presence of sodium borohydride $\left(\mathrm{NaBH}_{4}\right)$. PNP is a common organic pollutant, widely present in effluents of industries like dye production, pharmaceutical industries, fertiliser industries, leather tanning units and agricultural runoffs. A simple and fast remediation method of PNP contaminated water is selective hydrogenation of PNP to $p$-Aminophenol (PAP) by $\mathrm{NaBH}_{4}$. In many earlier reports, the fabrication of a stable reusable catalyst for PNP reduction usually undergoes a complex set of procedures $[20,21,22]$ or PNP reduction requires photocatalytic stimulus along with the nanoparticles $[23,24]$. Furthermore, recovery of catalysts is a major step in heterogeneous catalysis. Many reports have shown photocatalytic reduction of PNP in an overall liquid system where recovery of the catalyst is not possible $[25,26,27]$. The present study tackles these three aspects with a simple and comparatively cheap method of producing biomass-nanoparticle composite, efficient reduction of PNP without requiring light or any other reagent and easy recovery of the catalyst for reuse.

We also investigated the antimicrobial activity of the material and found it very efficient against both bacteria and fungi. Microbial strains used for the study comprised of two gram positive bacteria, Staphylococcus aureus and Streptococcus pyogenes, two gram negative bacteria, Vibrio cholerae and Escherichia coli; and three fungal strains, Penicillium citrinum, Aspergillus flavus and Candida ablicans.

This study demonstrates an integrated approach for utilisation of biomass produced during $\mathrm{CO}_{2}$ sequestration at large scale can be used for development of reusable and antimicrobial biomass-silver nanoparticles composite for catalytic reduction of p-Nitrophenol.

\section{Materials And Methods \\ 2.1. Chemicals}

The chemicals used in this study were procured from Himedia Pvt Ltd, India and were of analytical grade. These were sodium hydroxide $(\mathrm{NaOH})$ silver nitrate $\left(\mathrm{AgNO}_{3}\right)$, sodium borohydride $\left(\mathrm{NaBH}_{4}\right)$ and $\mathrm{p}$ Nitrophenol (PNP). Urea (46:0:0) and N:P:K (10:26:26) were procured from Indian Farmers Fertilizers Cooperative Limited (IFFCO), India and Paradip Phosphate Limited (PPL), India, respectively.

\subsection{Synthesis of silver nanoparticles-biomass composite material}

Microalga Scenedesmus sp. (IMMTCC-25) was cultivated in open raceway pond of CSIR-IMMT under the sunlight using low cost media $\left(0.1 \mathrm{gL}^{-1}\right.$ urea and $\left.0.75 \mathrm{gL}^{-1} \mathrm{NPK}\right)$ with $0.0075 \mathrm{M} \mathrm{NaOH}$ for purpose of $\mathrm{CO}_{2}$ gas sequestration. Raceway pond was run at $30,000 \mathrm{~L}$ capacity. The $\mathrm{CO}_{2}$ gas (20\% mixed with air) was infused through the algal culture and $\mathrm{NaOH}$ was used as intermediate $\mathrm{CO}_{2}$ sequester in medium. Later the algae utilized this $\mathrm{CO}_{2}$ for photosynthetic growth in raceway pond, thereby converting it into biomass. Biomass generated was a byproduct (or waste?) of the micro-algal $\mathrm{CO} 2$ sequestration process. Biomass was harvested after 15 days of cultivation, dried under sunlight and stored till further use. 
For the synthesis of silver nanoparticles, $1 \mathrm{M}$ stock solution of silver nitrate $\left(\mathrm{AgNO}_{3}\right)$ was prepared in deionised water. About $10 \mathrm{~g}$ dry algal biomass was soaked overnight in $100 \mathrm{~mL}$ deionised water. The soaked biomass was washed twice and resuspended in $1 \mathrm{~L}$ deionised water. The stock solution of $\mathrm{AgNO}_{3}$ was added to the biomass to obtain a final concentration of $10 \mathrm{mM} \mathrm{AgNO}_{3}$. The suspension was kept at room temperature under constant stirring for 72 hours. To monitor the bio-reduction of silver ions, $5 \mathrm{~mL}$ aliquot of the reaction solution was sampled at different time intervals. The concentration of silver in the supernatant was analysed by inductively coupled plasma-optical emission spectrometer (ICP-OES; Perkin Elmer Optima 2100 DV) after appropriate dilution of the sample. In the control experiment, $\mathrm{AgNO}_{3}$ was not added. Experiments were carried out in triplicates. After completion of the reaction, biomass was separated by filtration, washed with deionised water to remove unbound silver ions, if any, and dried in hot air oven at $50^{\circ} \mathrm{C}$. The dried AgNP-bound biomass material (biomass-AgNP) was used for further studies. The AgNPs were fixed onto the biomass by subjecting the material to calcination in a muffle furnace at different temperatures. Aliquots of $2 \mathrm{~g}$ of material were calcined at temperatures of $200^{\circ} \mathrm{C}$, $300^{\circ} \mathrm{C}, 400^{\circ} \mathrm{C}, 500^{\circ} \mathrm{C}$ and $600^{\circ} \mathrm{C}$.

\subsection{Characterisation of Biomass-AgNP material}

Field emission-scanning electron microscopy (FESEM; Carl Zeiss, Supra Gemini55) images of $\mathrm{AgNO}_{3}{ }^{-}$ exposed biomass were obtained to observe the morphology of the cells and the formation of AgNPs. The elemental composition of the sample was determined by EDS attached to the FESEM. Thermogravimetric analysis (TGA) was performed using a Thermogravimetric-Differential thermal analyzer (Mettler Toledo, TGA SDTA 851e). About 15-20 mg of $\mathrm{AgNO}_{3}$-exposed biomass was heated to $1000^{\circ} \mathrm{C}$ at the rate of 10 ${ }^{\circ} \mathrm{C} \min ^{-1}$ in aluminium oxide crucibles under nitrogen (99.9 wt\% purity) atmosphere. X-ray diffraction (XRD) was carried out using X-ray diffractomemter (Panalytical, XpertPro) having CuKa ( $k=1.54 \AA$ ) radiation and a programmable divergence slit. AgNP-biomass materials calcined at different temperatures were used for the analysis. The moisture and ash content of the unexposed dry biomass, uncalcined $\mathrm{AgNO}_{3}$-exposed biomass and calcined $\mathrm{AgNO}_{3}$-exposed biomass were analysed.

\subsection{Reduction of p-Nitrophenol}

To a solution of $10 \mathrm{ppm}$ p-Nitrophenol, $5 \mathrm{mM}$ sodium borohydride was added in the ratio 1: 0.1 to form a yellow solution of p-Nitrophenolate. Calcined biomass-AgNP composite material (CB- AgNP) was added to the solution and stirred constantly for 15 minutes. Absorbance was measured at regular intervals using a UV-Visible spectrophotometer (Cecil 7400) between 300 to $600 \mathrm{~nm}$. To optimize the amount of CBAgNP material required for maximum reduction of PNP, the above reaction was carried out using different amounts of CB-AgNP ranging from $1 \mathrm{mg} \mathrm{mL}^{-1}$ to $8 \mathrm{mg} \mathrm{mL}^{-1}$. Similarly, to determine the optimum calcination condition, materials calcined at different temperatures were used to carry out PNP reduction in the above manner. Reusability of the material was evaluated for 10 continuous cycles of reduction reactions. For this, the CB-AgNP material was recovered after each cycle of reduction by centrifugation and washed with distilled water before using in the next cycle. In another study of reuse, the material was kept suspended overnight in distilled water after five continuous cycles and then reused for additional five 
cycles. All results were interpreted with respect to the percentage of PNP reduced within 15 minutes of reaction.

\subsection{Antimicrobial tests}

The antimicrobial activity of CB-AgNP material was determined using well diffusion method. Six pathogenic microorganisms were used viz. two gram positive bacteria, Staphylococcus aureus and Streptococcus pyogenes; two gram negative bacteria, Vibrio cholerae and Escherichia coli; and three fungal strains, Penicillium citrinum, Aspergillus flavus and Candida ablicans. Nutrient agar plates and potato dextrose agar plates were used for bacteria and fungi, respectively. Plates were inoculated with small amount of respective microorganisms using spread plate technique. Three wells were punched in each plate using sterile tips. CB-AgNP material was added in three different amounts in each plate, i.e. 10 $\mathrm{mg}, 20 \mathrm{mg}$ and $50 \mathrm{mg}$. After incubation for 24 hours, diameter of the inhibition zone was measured.

\section{Result And Discussion}

\subsection{Synthesis and characterisation of the CB-AgNP material}

For this study the microalgal biomass generated as waste or byproduct during micro-algal $\mathrm{CO}_{2}$ sequestration process was used. Biomass was obtained after large scale 30,000 L cultivation in open raceway pond under sunlight. Scenedesmus biomass was harvested, dried and stored under room temperature. Throughout the storage period the biomass retained its green colour. When exposed to silver nitrate solution, the colour of biomass changed from green to brown. This might be due to the conversion of $\mathrm{Ag}^{+}$ions to $\mathrm{Ag}^{0}$ within the biomass. The control solution that did not have silver nitrate showed no visible colour change. Also while recovering the exposed biomass by filtration, the filtrate appeared colourless. This further showed that the synthesized AgNPs adhered to the biomass and did not diffuse out into the solution.

ICP-OES study of the filtrate showed that uptake of $\mathrm{Ag}^{+}$ions by the biomass was instantaneous. About $85 \%$ of $\mathrm{Ag}^{+}$ions were taken up by the biomass within five minutes of addition after which $\mathrm{Ag}^{+}$ concentration in the solution remained constant in the solution (Fig. 1a). However, a gradual change in colour of the biomass from green to brown was observed between 48-72 hours of exposure. This indicated that while $\mathrm{Ag}^{+}$ions were adsorbed onto the biomass surface instantaneously, the formation of $\mathrm{Ag}^{0}$ within the biomass was a gradual and slow process. In a similar study of the synthesis of silver nanoparticles using live Scenedesmus biomass culture, exposure time was optimised to 72 hours [19].

FESEM micrograph (Fig. 1b, 1c) of the $\mathrm{AgNO}_{3}$ exposed biomass showed the formation of silver nanoparticles. It revealed that most of the cells were still intact retaining their oval shape and measured roughly $10 \mu \mathrm{m}$. White spots on the cells represented AgNPs, distributed throughout the cell. EDAX of typical biomass-AgNP composite showed signals for silver with additional peaks of $\mathrm{C}, \mathrm{O}, \mathrm{Na}$, Si and P (Fig. 1d). The ash content analysis showed the presence of ash as high as $41.19 \%$ in the unexposed 
biomass. As the biomass was cultured in open raceway pond, this could be due to dust entering in medium and ultimately landing onto the biomass. As expected, the moisture content of the calcined $\mathrm{AgNO}_{3}$-exposed biomass was lower and ash content was higher than its uncalcined counterpart (Table 1).

Table 1

Moisture and ash content in $\mathrm{AgNO}_{3}$-unexposed, exposed and calcined biomass.

\begin{tabular}{|lll|}
\hline Sample & Moisture (\%) & Ash (\%) \\
\hline Unexposed Biomass & 17.20 & 41.19 \\
\hline Silver Exposed Biomass & 17.59 & 45.62 \\
\hline Calcined Silver Exposed Biomass & 2.37 & 96.51 \\
\hline
\end{tabular}

TGA-DTA of $\mathrm{AgNO}_{3}$-exposed biomass showed the characteristic pattern of weight loss for Scenedesmus biomass (Fig. 2a). Corresponding to the pattern previously reported by Jena et al [19], three stages of weight loss were observed. Initial weight loss below $200^{\circ} \mathrm{C}$ may be assigned to the evaporation of free and bound water molecules. The second stage of weight loss observed between $200^{\circ} \mathrm{C}$ to $400{ }^{\circ} \mathrm{C}$ may be assigned to decomposition of proteins and carbohydrates. In the final stage, decomposition of organic matter occurs beyond $400^{\circ} \mathrm{C}$. Maximum weight loss was observed in the first stage owing to high moisture. Broadening of the second peak could be due to alteration in thermal stability of proteins due to binding of silver nanoparticles. In the third stage, maximum weight loss was observed which prolonged from $600^{\circ} \mathrm{C}$ till $750^{\circ} \mathrm{C}$. This may be due to silver nanoparticles resisting weight loss at higher temperatures as metals are known to have a slower rate of weight loss. Dust particles present in biomass (due to open raceway pond cultivation) also resists weight loss and may also be another contributing factor in the same. Also, weight loss at the third stage was lower than other stages. This could be due to lower lipid content in the biomass.

$\mathrm{XRD}$ of the biomass-AgNP material revealed the nature of uncalcined ( $\mathrm{AgNO}_{3}$-exposed dried biomass) and calcined composite (Fig. 2b). The XRD peaks appeared more prominent with an increase in calcination temperature. The spectrum of the calcined materials, especially when calcined at $400{ }^{\circ} \mathrm{C}$ or above, showed Bragg reflection peaks at $2 \theta=38.48^{\circ}, 44^{\circ}, 64.74^{\circ}$ and $77.4^{\circ}$. These peaks correspond to the fcc structure of the AgNPs at lattice planes of (111), (200), (220) and (311). For signal peaks to be distinct in the XRD spectrum, the analyte must constitute at least $5 \%$ of the sample. The revelation of the Ag peaks in materials calcined at higher temperatures was supportive of the above fact. Calcination led to the decomposition, oxidation and loss of organic content of the biomass, as evident from the TGA data, which increased the relative amount of metallic AgNPs in the sample. So as the temperature of calcination increased, diffraction peaks became more prominent. Additional peaks were those of $\mathrm{Ag}_{2} \mathrm{O}$, resulting out of oxidation of Ag within the material. Another high-intensity peak observed was that of $\mathrm{SiO}_{2}$. Though small amount of $\mathrm{Si}$ is necessary for microalgal growth, presence of high amount of it could 
be attributed to silicates of dust particles getting into biomass as it was cultivated in open raceway pond. The optical images of the calcined materials showed change in colour with increasing calcination temperature (Fig. 2C). The initial green colour of the biomass disappeared with gradual decomposition of chlorophyll pigments. As the temperature reached $400{ }^{\circ} \mathrm{C}$, the biomass turned black due to charring. Further heating led to reduction of the biomass to a lighter colored ash.

\subsection{Reduction of p-Nitrophenol}

The catalytic efficiency of the calcined-biomass-AgNP composite material (CB-AgNP) was evaluated in reduction of PNP in presence of $\mathrm{NaBH}_{4}$ as reducing agent. The reduction reaction takes place in two steps. In the first step, $\mathrm{PNP}$ is converted to $\mathrm{p}$-Nitrophenolate $\left(\mathrm{PNP}^{-}\right)$ion by the addition of $\mathrm{NaBH}_{4}$. This reaction is spontaneous and can be observed visually as the straw yellow colour of PNP solution changes to a bright yellow $\mathrm{PNP}^{-}$solution. In the next step, hydrogenation of $\mathrm{PNP}^{-}$takes place to form a colourless solution of PAP. However this reaction is thermodynamically unfavorable and requires a suitable catalyst. The catalyst relays the electrons from $\mathrm{BH}_{4}{ }^{-}$to $\mathrm{PNP}^{-}$and reduces the later to $\mathrm{p}$ Aminophenolate (PAP). A solution of PAP is colourless. Silver nanoparticles (AgNPs) were used as a catalyst by Pradhan et al. [28] for reduction of PNP where it was shown that small growing silver nanoparticles acted as nanoelectrodes efficiently transmitting electrons from $\mathrm{BH}_{4}{ }^{-}$for reduction of PNP to PAP [28]. While working with a heterogeneous catalysis system many reports have proposed a fourstep process for catalysis of $\mathrm{NaBH}_{4}$ mediated reduction of PNP [21, 29, 30]. Kong et al. [31] have provided further insights into the mechanism of the reduction process.

In the present study, when $\mathrm{NaBH}_{4}$ was added to PNP solution, an alkaline condition prevailed and PNP was converted to $\mathrm{p}$-Nitrophenolate $\left(\mathrm{PNP}^{-}\right)$. This reaction was observed visually as the straw yellow colour of PNP solution changed to a bright yellow $\mathrm{PNP}^{-}$solution. $\mathrm{PNP}^{-}$had an absorption maxima at $400 \mathrm{~nm}$, as observed with UV-Visible spectroscopy. CB-AgNP composite material (calcined at $400{ }^{\circ} \mathrm{C}$ ) could catalyse the reduction of $80 \%$ of PNP $^{-}$within one minute of addition (Fig. 3b). When left under constant stirring, more than $90 \%$ reduction was achieved within 15 minutes. The progress of the reaction was monitored by using UV-Visible spectroscopy (Fig. 3a). As the reaction advanced, the characteristic peak of $\mathrm{PNP}^{-}$at $400 \mathrm{~nm}$ decreased while at around $300 \mathrm{~nm}$ a new peak appeared representing formation of PAP. The yellow colour of the solution faded gradually and completely bleached at the end of the reaction (Fig. 3c). The reduction reaction is depicted in Fig. 3d.

\subsection{Effect of Calcination Temperature}

Calcination was an important step in developing the CB-AgNP composite material as a catalyst. It fixed the AgNPs onto the biomass and prevented its decaying due to presence of moisture. To understand how calcination affected the catalytic activity of the material a series of experiments were carried out while varying the calcination temperature. As calcination is an energy consuming process, these experiments helped in determining an optimum temperature (as low as possible) for calcination so as to ensure maximum catalytic efficiency while conserving as much energy as possible. Effect of calcination 
temperature on the percentage of PNP reduction was analysed (Fig. 4a). The uncalcined biomass showed poor catalytic activity probably due to the covering of biomaterials on the AgNPs making the reaction slower. It could reduce only about $30 \% \mathrm{PNP}$ in the solution. With calcination, a significant increase in PNP reduction was observed. The composite material calcined at 200 and $300{ }^{\circ} \mathrm{C}$ reduced about $70 \%$ of PNP. With an increase in calcination temperature further to $400{ }^{\circ} \mathrm{C}$, reduction increased further to $90 \%$. Thereafter, it remained almost constant upto temperature of $600{ }^{\circ} \mathrm{C}$. This also indicated that AgNPs were bound to cellular scaffolds in the biomass that remained thermally stable till $600{ }^{\circ} \mathrm{C}$. Calcining the material beyond it led to the decomposition of scaffolds, causing aggregation and fusion of silver nanoparticles, as evident from TGA-DTA data. Beyond that temperature, decline in the catalytic efficiency of the material was observed. As higher temperature requires higher energy inputs, $400{ }^{\circ} \mathrm{C}$ was considered as optimum and for all further experiments CB-AgNP material calcined at $400{ }^{\circ} \mathrm{C}$ was used. In the subsequent study, the effect of load of CB-AgNP material (calcined at $400^{\circ} \mathrm{C}$ ) on the reduction of PNP- was investigated (Fig. 4b). Even with as low as $1 \mathrm{mg} \mathrm{mL}^{-1}$ of CB-AgNP, more than $50 \%$ reduction was observed. Evidently, with an increase in the load of CB-AgNP material from $1 \mathrm{mg} \mathrm{mL}^{-1}$ to $8 \mathrm{mg} \mathrm{mL}^{-1}$, the percentage of PNP reduction increased gradually till $5 \mathrm{mg} \mathrm{mL}^{-1}$ and remained almost constant thereof. At $5 \mathrm{mg} \mathrm{mL}^{-1}$ and $8 \mathrm{mg} \mathrm{mL}^{-1}$, the material could reduce above $90 \%$ of PNP in the solution.

\subsection{Reusability test of the CB-AgNP material}

The advantage of heterogeneous catalysis is the reusability of the catalyst. Hence, the reusability of the CB-AgNP material in PNP reduction was also evaluated (Fig. 4c and $4 \mathrm{~d}$ ). The material calcined at $400{ }^{\circ} \mathrm{C}$ was used for this study. When $5 \mathrm{mg} \mathrm{mL}^{-1}$ of the material was used for ten cycles of reactions continuously, PNP reduction declined gradually with each cycle. In the first cycle, the percentage of PNP reduced was as high as $95 \%$, but it fell significantly to $40 \%$ by the sixth cycle. In another set of experiment, the material was used for five cycles of reduction, left suspended in distilled water overnight and reused again for another five cycles. Again PNP reduction declined gradually to around $45 \%$ in the first five cycles. Interestingly, when it was reused after overnight suspension in water, the catalytic activity of the material seemed to have recovered and PNP reduction increased to $60 \%$. One of the important steps in heterogeneous catalysis is the adsorption of the substrates on to the catalyst surface. In the present case, $\mathrm{p}$-Nitrophenolate and $\mathrm{BH}_{4}$ might be getting adsorbed on to the $\mathrm{CB}-\mathrm{AgNP}$ surface and diffused to the catalytic sites where AgNPs facilitated the transfer of electrons from $\mathrm{BH}_{4}{ }^{-}$to $\mathrm{p}$ Nitrophenolate, simultaneously reducing it to the final product, $p$-Aminophenol (PAP). PAP molecules then get desorbed from the CB-AgNP surface and released into the solution. The efficacy of the material depended on the availability of surface area for PNP $^{-}$molecules to be adsorbed which subsequently depended on removal of p-Aminophenol molecules from the surface. The decrease in PNP reduction may be attributed to the loss of AgNPs in between cycles and/or the blockage of biomass surface and its catalytic sites by $\mathrm{p}$-Aminophenol molecules. The later phenomenon explains the recovery of the catalytic property of the material when subjected to an overnight water wash. Suspending the material in water might have facilitated the removal of p-Aminophenol from the surface. However, the material recovered only to degrade $60 \%$ of PNP as opposed to $95 \%$ at the beginning, which might be due to the loss of 
AgNPs in between cycles/washing with water. Similarly, a gradual decline in the rate of reaction was observed for subsequent cycles of reduction. In the beginning, the rate of reaction was observed to be around $0.60 \mathrm{mg} \mathrm{mL}^{-1} \mathrm{~min}^{-1}$ which declined to $0.30 \mathrm{mg} \mathrm{mL}^{-1} \mathrm{~min}^{-1}$ at the fifth cycle. This observation was coherent with the decline in the percentage of PNP reduction to half of the initial value within five cycles of continuous reuse. If reused continuously without break, the rate further decreased to $0.20 \mathrm{mg}$ $\mathrm{mL}^{-1} \mathrm{~min}^{-1}$ by completion of ten cycles. But with an intermittent water wash after five cycles, the rate of reaction increased in the sixth cycle to $0.35 \mathrm{mg} \mathrm{mL}^{-1} \mathrm{~min}^{-1}$ and remained at about $0.30 \mathrm{mg} \mathrm{mL}^{-1} \mathrm{~min}^{-}$ ${ }^{1}$ after ten cycles. The decline in the rate of reaction suggests an adsorption mediated catalysis process. A possible mechanism constituting four basic steps of the adsorption-mediated, CB-AgNP catalysed reduction of PNP to PAP is shown in Fig. 5.

\subsection{Antimicrobial tests}

The antimicrobial activity of the CB-AgNP material is illustrated in Fig. 6. Measurement of zone of inhibition (Zol) shows that the material confers decent activity against both bacterial and fungal strains. The activity increased with increase in the material load in each microbial plate. At $10 \mathrm{mg}$ load, the material showed comparable activity against all strains of microorganism. However as the material load was increased to $50 \mathrm{mg}$, distinctive activity was observed against fungal strains. The Zol measurements are shown in Table 2.

Table 2

Zone of inhibition of antimicrobial tests using CB-AgNP material

\begin{tabular}{|lllll|}
\hline Microbial Strain & & \multicolumn{3}{c|}{ Inhibition Zone (cm) } \\
\cline { 3 - 5 } & & CB-AgNP & CB-AgNP & CB-AgNP \\
Gram -ve Bacteria & Escherichia coli & $\mathbf{( 1 0} \mathbf{~ m g})$ & $\mathbf{( 2 0} \mathbf{~ m g})$ & $\mathbf{( 5 0} \mathbf{~ m g})$ \\
\cline { 2 - 5 } & Vibrio cholerae & $1.72 \pm 0.05$ & $1.85 \pm 0.17$ & $1.97 \pm 0.12$ \\
Gram + ve Bacteria & Streptococcus pyogenes & $1.42 \pm 0.05$ & $1.45 \pm 0.06$ & $1.72 \pm 0.09$ \\
\cline { 2 - 5 } & Staphylococcus aureus & $1.48 \pm 0.05$ & $1.52 \pm 0.05$ & $1.95 \pm 0.06$ \\
\cline { 2 - 5 } Fungi & Penicillium citrinum (UC1F1) & $2.27 \pm 0.09$ & $2.62 \pm 0.05$ & $3.20 \pm 0.12$ \\
\cline { 2 - 5 } & Aspergillus flavus (BMF4) & $1.32 \pm 0.10$ & $2.17 \pm 0.05$ & $2.92 \pm 0.05$ \\
\cline { 2 - 5 } & Candida ablicans & $1.48 \pm 0.09$ & $1.84 \pm 0.08$ & $2.28 \pm 0.04$ \\
\hline
\end{tabular}

The mechanism by which AgNPs confer molecular toxicity remains highly debated till date. Some authors report particle-specific activity of silver nanoparticles where the size, shape and surface charge and functional ligands on the nanoparticles are said to be the responsible factors[32]. Whereas other reports sustain that release of silver ions from the nanoparticle core upon oxidation is necessary for their antimicrobial activity [33]. In this study the silver nanoparticles remain bound to Scenedesmus biomass 
and XRD pattern shows the presence of both metallic and oxidized Ag. Though the material was found to be an efficient antimicrobial agent, it could not be determined whether AgNPs or Ag + ions were responsible for it.

\section{Conclusion}

We discovered that dry microalgae biomass harvested during $\mathrm{CO}_{2}$ sequestration as byproduct of the process can be used to synthesize silver nanoparticles with catalytic and antimicrobial properties. The silver nanoparticle-immobilised biomass could efficiently catalyse the reduction of PNP in aqueous condition without requiring light or any other reagent. As the CB-AgNP was a solid phase catalyst, it could be recovered and reused efficiently with intermittent breaks between consecutive cycles of reduction. $A$ continuous system with CB-AgNP material packed column can be developed in the future for reduction of p-Nitrophenol. The reduction and removal of p-Nitrophenol hold both environmental and industrial significance. Hence this material is an efficient and economic source for p-Nitrophenol reduction. The material was also found to be a potent antimicrobial agent with activity against gram positive and gram negative bacteria as well as pathogenic fungi. In the quest for development of novel antimicrobial products, the CB-AgNP composite can singularly prove to be an efficient alternative as a component in exterior coatings in buildings and establishments for maintaining an aseptic environment/ topical applications. This study also demonstrates the potential of microalgae biomass-silver nanoparticle in development of an eco-friendly and sustainable integrated system targeted towards $\mathrm{CO}_{2}$ sequestration, industrial p-Nitrophenol reduction and microbial disinfection.

\section{Declarations}

\section{Acknowledgement}

All authors are thankful to Director CSIR-IMMT, Bhubaneswar for permission to publish this article. NP would like to thank the financial support of Department of Science and Technology, Government of India (Grant Number DST/IS-STAC/CO ${ }_{2}$-SR-169/13(G) and DST-UKIERI Award No. DST/INT/UK/P-128/2016). SSP would like to thank Council of Scientific and Industrial Research, Govt. of India for the fellowship under CSIR-JRF Scheme (Grant Number 20/12/2015 (ii) EU-V).

\section{Conflict of interest}

The authors declare that they have no conflict of interest.

\section{References}

[1] A. Gupta, M. Tandon \& A. Kaur. Nanotechnol. Environ. Eng. 5, 27 (2020). https://doi.org/10.1007/s41204-020-00092-y 
[2] M. Shah, D. Fawcett, S. Sharma, S.K. Tripathy \& G.E.J. Poinern. Materials (Basel); 8(11) (2015): 72787308. DOI: $10.3390 / \mathrm{ma} 8115377$

[3] H. Duan, D. Wang \& Y. Li. Chem. Soc. Rev. 44 (2015) 5778-5792.

[4] S.A. Dahoumane, M. Mechouet, K. Wijesekera, C.D.M. Filipe, C. Sicard, D. Bazylinski \& C. Jeffryes .Green Chem. 19 (2017) 552-587

[5] M.I. Khan, J.H. Shin \& J.D. Kim. Microb. Cell. Fact. 17 (2018), 36. https://doi.org/10.1186/s12934-0180879-x

[6] P. Das, S.S. Aziz, \& J. Obbard. Two phase microalgae growth in the open system for enhanced lipid productivity. Renewable Energy, 36 (2011) 2524-2528.

[7] F. Arsiya, M.H. Sayadi, \& S. Sobhani. J. Water. Environ. Nanotechnol. 2(3) (2017) $166 \rrbracket 173$.

[8] J. Jena, N. Pradhan, V. Aishvarya, R.R. Nayak, R, B.P. Dash, L.B. Sukla, P.K. Panda \& B.K. Mishra. J. Appl. Phycol. 27 (2014) $2251 \rrbracket 2260$

[9] P. Khanna, A. Kaur \& D. Goyal. J. Microbiol. Methods. 163 (2019) 105656. DOIdoi.org/10.1016/j.mimet.2019.105656

[10] N. Hanagata, T. Takeuchi, Y. Fukuju, D.J. Barnes \& I. Karube. Phytochemistry, 31(10) (1992) 3345 \& 3348. DOI: 10.1016/0031-9422(92)83682-0

[11] J. Jena, M. Nayak, H.S. Panda, N. Pradhan, C. Sarika, P\&a, P., V.S.K. Rao, B.N. Prasad \& L.B. Shukla. World Environment, 2(1), (2012) $11 \otimes 16$. DOI:10.5923/J.ENV.20120201.03

[12] G. Rhee. J. Phycol, 9 (1973) 495-506. DOI: 10.1111/j.1529-8817.1973.tb04126.x

[13] S. Basu, A.S. Roy, K. Mohanty, \& A.K. Ghoshal. Bioresour Technol. 143 (2013) 369-377. DOI: 10.1016/j.biortech.2013.06.010

[14] S.H. Ho, W.M. Chen \& J.S. Chang. Bioresour. Technol. 101(22) (2010) 8725-8730. DOI: 10.1016/j.biortech.2010.06.112

[15] M. Nayak, S.S. Rath, M. Thirunavoukkarasu, P.K. Panda, B.K. Mishra \& R.C. Mohanty. J. Microbiol. Biotechnol. 28, 23(9) (2013) 1260-1268. DOI: 10.4014/jmb.1302.02044.

[16] N. Pradhan, \& B. Das. Recent Advancements in Biofuels \& Bioenergy Utilization. Springer, Singapore (2018) $285 \otimes 302$.

[17] D. Tang, W. Han, P. Li, X. Miao \& J. Zhong. Bioresour. Technol. 102(3) (2011) $3071 \rrbracket 3076$. 
[18] N. Aziz, T. Fatma, A. Varma \& R. Prasad. Journal of Nanoparticles (2014), http://dx.doi.org/10.1155/2014/689419

[19] J. Jena, N. Pradhan, R. Nayak, B.P. Dash, L.B. Sukla, P.K. Panda \& B.K. Mishra. J. Microbiol. Biotechnol. 24(4) (2014) $522 \otimes 533$.

[20] J.R. Chiou, B.H. Lai, K.C. Hsu \& D.H. Chen. J. Hazard. Mater. 248-249 (2013) 394 \400. DOI: 10.1016/j.jhazmat.2013.01.030.

[21] W. Fang, Y. Deng, L. Tang, G. Zeng, Y. Zhou, X. Xie, J. Wang, Y. Wang \& J. Wang. J. Colloid. Interface. Sci. 490(2017), $834 \otimes 843$.

[22] Z.H. Ren, H.T. Li, Q. Gao, H. Wang, B. Han \& K.S. Xia. Materials \& Design. 121 (2017) 167 175. 10.1016/j.matdes.2017.02.064

[23] S. Ahmed, M.G. Rasul, W.N. Martens, R. Brown \& M.A Hashib. Desalination, 261 (1ه 2) (2010) $3 \otimes 18$.

[24] L. Yang, S. Luo, Y. Li, Y. Xiao, Q. Kang \& Q. Cai. Environ. Sci. Technol. 44(19) (2010) $7641 \otimes 7646$.

[25] S. Francis, S. Joseph, E.P. Koshy \& B. Mathew. Environ. Sci. Pollut. Res. Int. 24(21) (2017) 1734717357. DOI: 10.1007/s11356-017-9329-2.

[26] P.S. Rajegaonkar, B.A. Deshpande, M.S. More, S.S. Waghmare, V.V. Sangawe \& A. Inamdar. Mater. Sci. Eng. C. 93(2018) $623 \otimes 629$.

[27] W. Zhang, F. Tan, W. Wang, X. Qiu, X. Qiao \& J. Chen. J. Hazard. Mater. $217 \otimes 218(2012) 36 \rrbracket 42$.

[28] N. Pradhan, A. Pal \& T. Pal. Colloids Surf. A. 196 (2002) $247 \otimes 257$.

[29] V.K. Gupta, N. Atar, M.L. Yola, Z. Üstündağ \& L. Uzun. Water Research, 48(2014), pp. $210 \otimes 217$.

[30] P. Guo, L. Tang, J. Tang, G. Zeng, B. Huang, H. Dong, Y. Zhang, Y. Zhou, Y. Deng, L. Ma \& S. Tan. Journal of Colloid Interface Science, 469(2016), pp. $78 \otimes 85$.

[31] X. Kong, H. Zhu, C. Chen, G. Huang \& Q. Chen. Chem. Phys. Lett. 684 (2017), $148 \otimes 152$.

[32] N. Duran, M. Duran, B. Jesus, A.B. Seabra, W.J. Favaro, \& G. Nakazato. Nanomedicine: Nanotechnology, Biology, \& Medicine, 12 (2016) 789 \ 799

[33] Z. Xiu, Q. Zhang, H.L. Puppala, V.L. Colvin \& J.J. Alvarez. Nano Letters. 12 (2012) $4271 \rrbracket 4275$

\section{Figures}




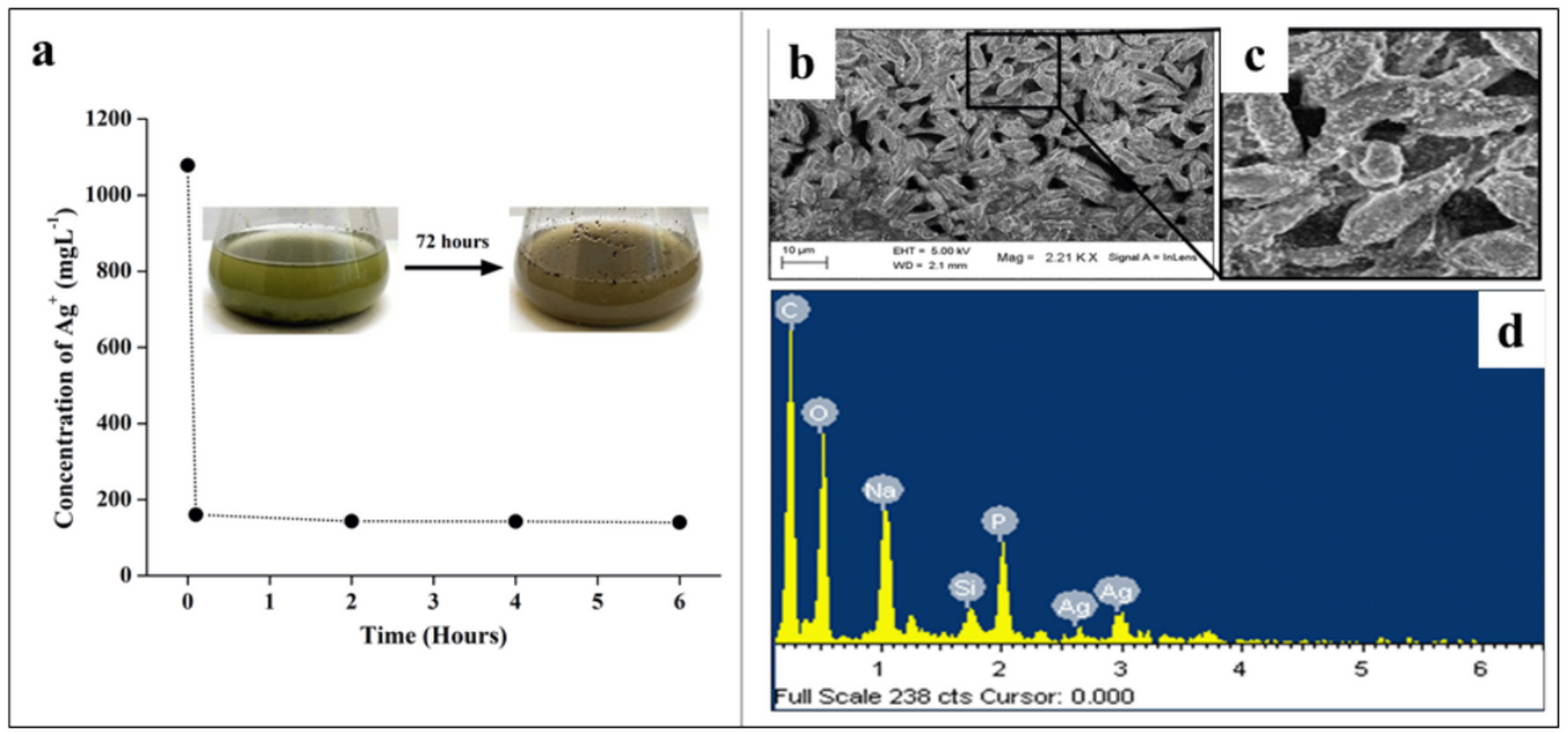

\section{Figure 1}

(a) Change in concentration of $\mathrm{Ag}+$ ions in the reaction solution with respect to time of exposure as determined by ICP-OES, inset shows the optical image of biomass solution before and after synthesis of silver nanoparticles. FESEM micrograph: (b and $c$ ) shows intact and broken cells of Scenedesmus with white spots representing silver nanoparticles, (d) EDAX spectrum of the biomass

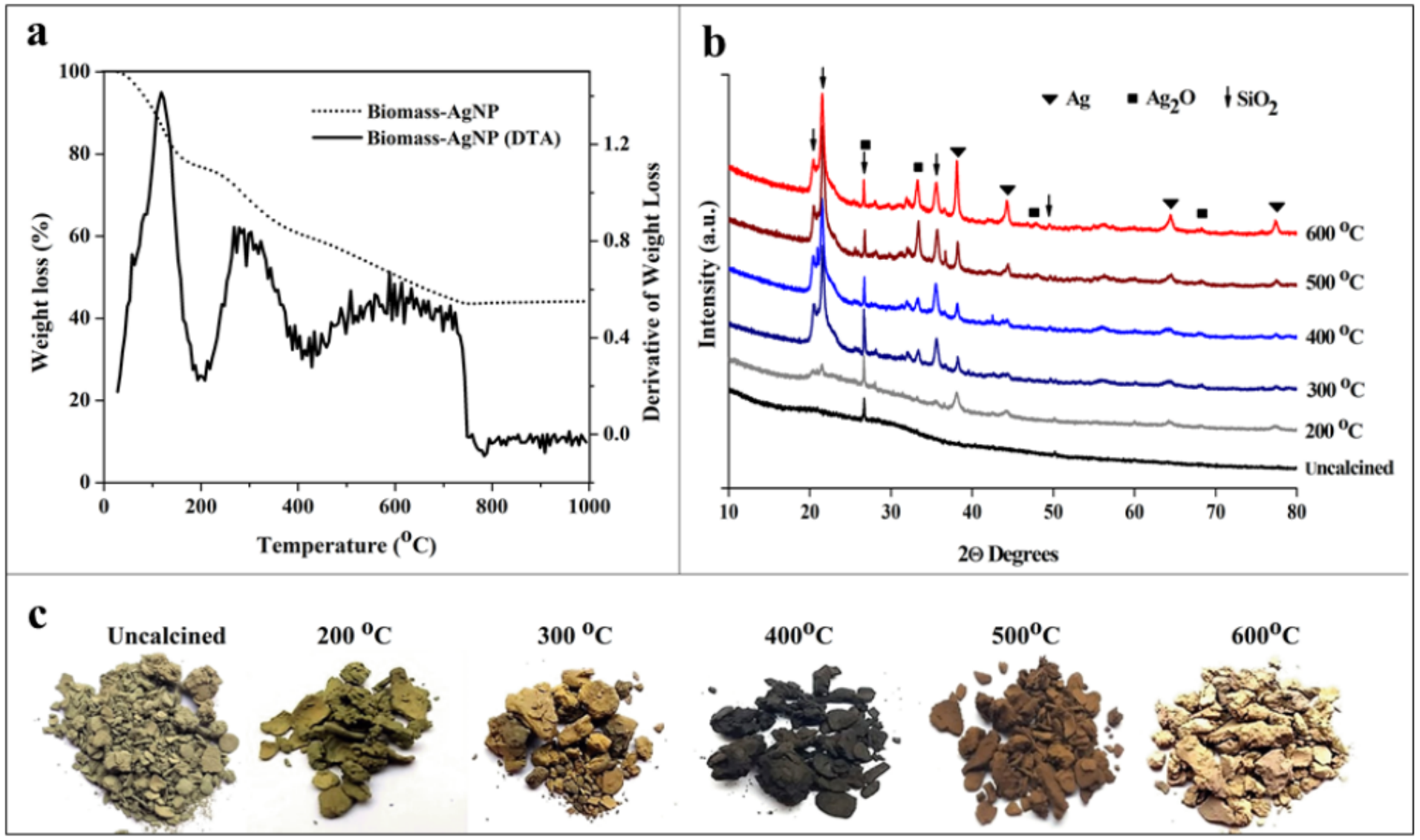


Figure 2

(a) TGA-DTA of AgNO3-exposed biomass (b) XRD pattern of AgNO3-exposed biomass calcined at different temperatures (c) Respective optical images of materials

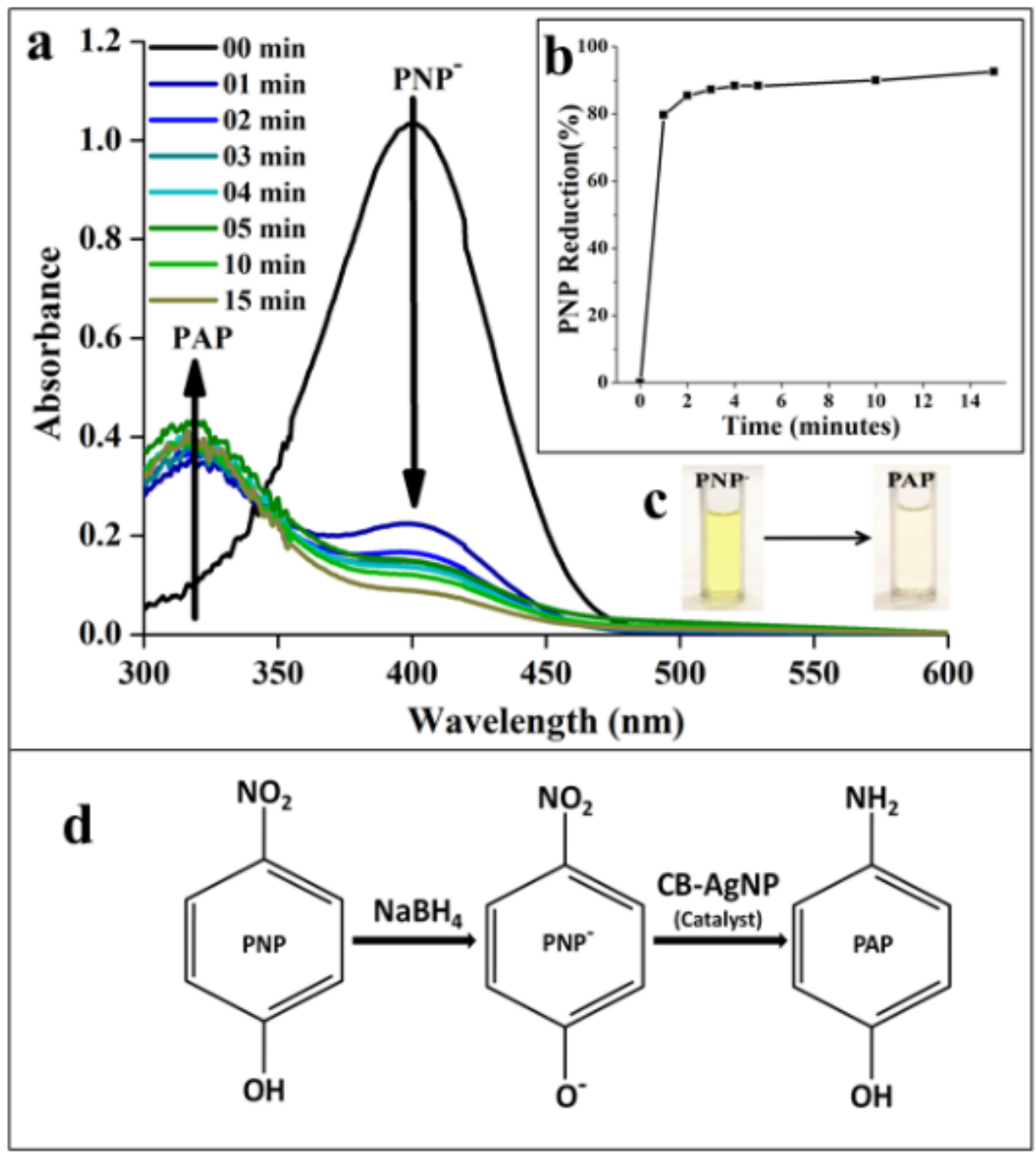

Figure 3

(a) UV-Visible spectra of PNP- reduction with CB -AgNP, (b) Percentage reduction vs. Time of reaction, (c) fading of yellow colour of PNP- to a colourless PAP solution after reduction, (d) the reduction reaction of PNP to PAP by $\mathrm{NaBH} 4$ in presence of $\mathrm{CB}-\mathrm{AgNP}$ 


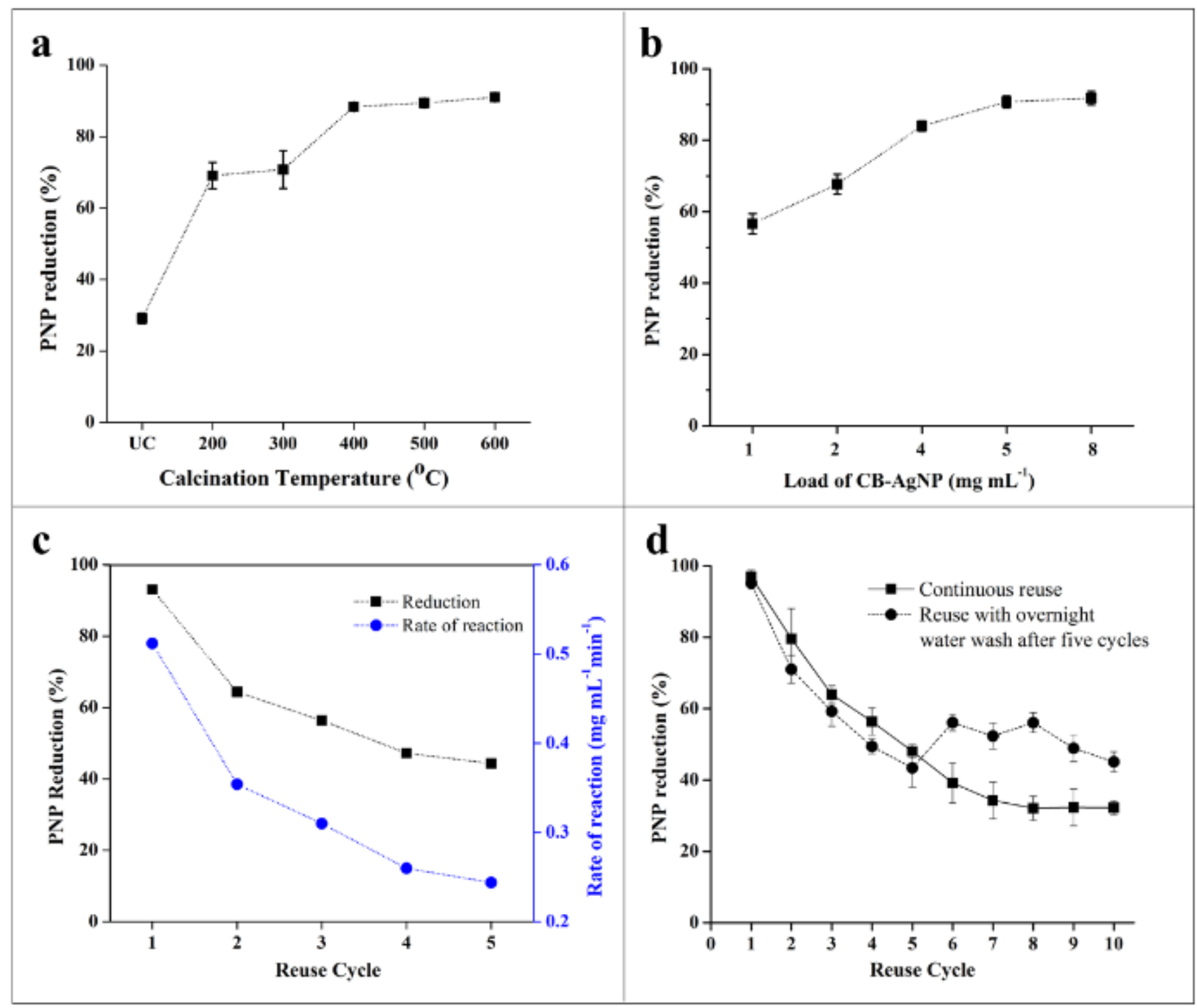

Figure 4

Catalytic efficiency of CB-AgNP material with respect to: (a) temperature of calcination and (b) load of the CB-AgNP material (c) reuse for five cycles (d) reuse for ten cycles with intermittent water wash 


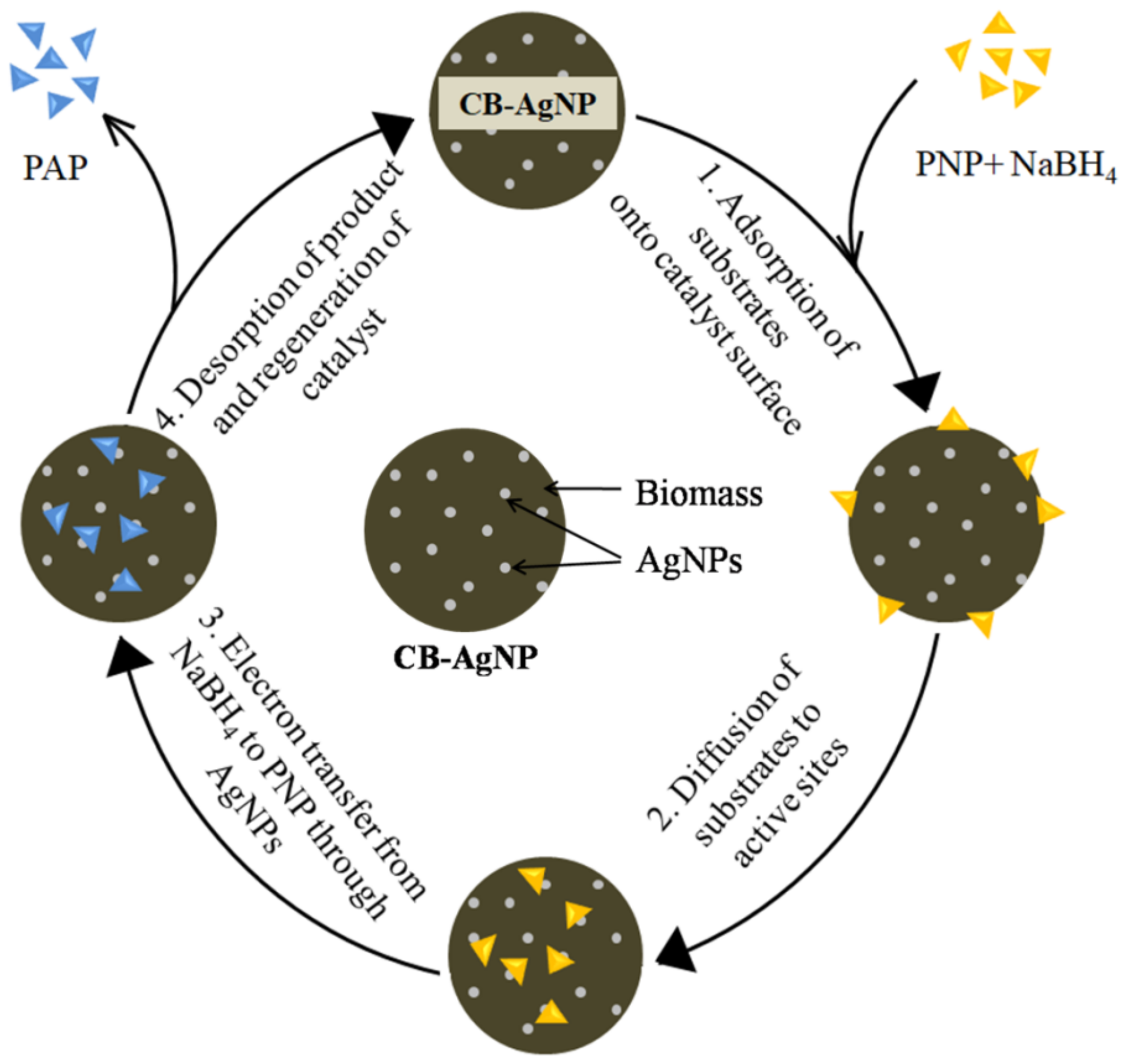

Figure 5

Possible mechanism of PNP reduction by $\mathrm{NaBH} 4$ in presence of $\mathrm{CB} \otimes \mathrm{AgNP}$ material
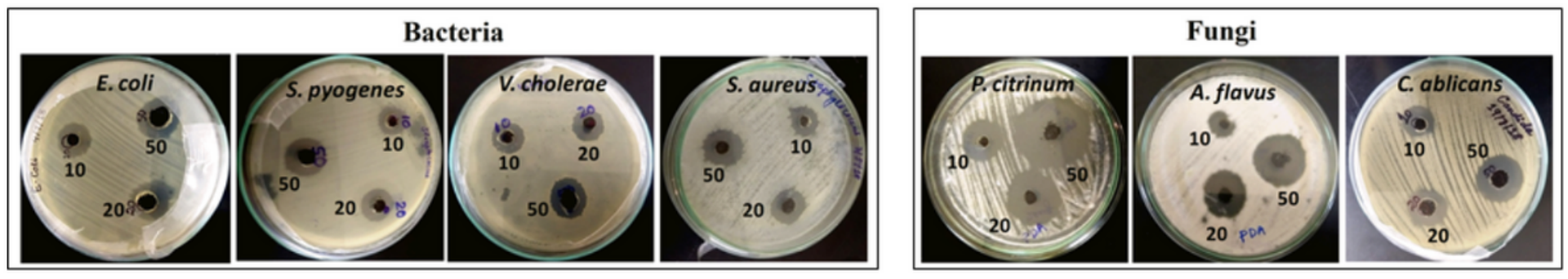

Figure 6 
Agar diffusion method showing zone of inhibitions of bacteria and fungi growth in presence of different loads of CB-AgNP material

\section{Supplementary Files}

This is a list of supplementary files associated with this preprint. Click to download.

- GRAPHICALABSTRACT.png 